\title{
Miniature Electron Beam Columns: From the Lab to the Field
}

\author{
Lawrence Muray ${ }^{1}$, Scott Davilla ${ }^{2}$ and James Spallas ${ }^{3}$ \\ 1,2,3. Keysight Technologies, Inc. Nano Scale Sciences Division, Santa Clara, CA, USA.
}

Scanning electron microscopes (SEMs) have been the workhorse of high-technology research and development for well over 50 years with applications ranging from the life sciences to forensic sciences. Improvements in SEM performance, operating conditions, ability to accommodate analytical instrumentation, portability, user interface, software, and automation, have progressed at a rapid rate bringing SEM into main stream usage. Today, innovations such as low-voltage imaging and compound or cathode lenses allow researchers to routinely image and analyze nanoscale structures at $<2 \mathrm{~nm}$ resolution [1]. However, the fundamental architecture of SEMs -- a high-brightness macro-scale electron source coupled with precision-machined magnetic and electrostatic lens, deflection and correction elements -- has also imposed, with few exceptions, the requirement that the sample be brought to the system rather than the system to the sample. Removing this constraint could create new types of field applications which would benefit from the SEM's high resolution imaging and analytical capabilities. An example from space exploration is shown in Fig 1, where undetected fine-grained lunar dust can create potentially life threatening situations [2].

Miniature electron beam columns, shown in Fig 2, are a relatively new class of electrostatic columns fabricated from silicon using standard microfabrication processes. The defining characteristics of these columns are a thermal field emission (TFE) source, low voltage operation (typically < $3 \mathrm{keV}$ ), simple design (two lenses, no crossover), aperture lenses, ceramic carriers and impedance-controlled patterned interconnects. Production versions of miniature columns achieve $<10 \mathrm{~nm}$ resolution at $1 \mathrm{keV}$, and have demonstrated $<6 \mathrm{~nm}$ resolution at higher beam energies [3]. Currently, they are available only in a desktop SEM, but, with the small size, low power and high performance, miniature columns are ideal candidates for field applications.

Development of the miniature columns is still in its infancy. There is plenty of room to improve or tailor the design to meet the requirements of field applications. Three major considerations are: a) column performance, b) addition of detectors, and c) system ruggedization. The latter is a critical requirement for portable applications, especially in extreme or harsh environments.

In order to enhance the capabilities of the columns, such as improvements to resolution, extended working distance, or extended voltage range, it is important to have a complete understanding the unique constraints arising from electrostatic lenses and miniaturization. These constraints typically have no counterparts in conventional SEM design. An example is trade-offs between bore diameter and manufacturing yield or trade-offs between resolution and beam current for high throughput or improved signal-to-noise [4].

With new detectors, the problem becomes one of overcoming tight space constraints, new operating conditions and/or increased working distance. An example of this is the recent unconventional integration of energy dispersive spectroscopy (EDS) with the miniature columns. Excitation energies $>10 \mathrm{keV}$ are necessary to obtain the unique the $\mathrm{x}$-ray signature of most materials, yet miniature columns currently cannot operate above a few $\mathrm{keV}$. This problem is overcome by biasing the sample to > 
$+13 \mathrm{keV}$, which creates post-lens acceleration and $15 \mathrm{keV}-20 \mathrm{keV}$ landing energies [5]. The result is good EDS performance (with a few limitations described elsewhere), and interesting new physics in the $\mathrm{X}$-ray generation process. Sample charging, for example, is substantially reduced even at high landing energies. This effect is assumed to be a result of the suppression of secondary and low energy electron emission by the large biasing fields.

Finally, creating a rugged, field-deployable SEM requires improvements in many of the existing system components, including use of radiation-hardened electronics, improved packaging, and better environmental isolation. Ruggedization of the miniature columns requires primarily improvements in the electron source stability, reliability, startup and recovery. The column itself is rigid by design, but must be evaluated for resilience to shock, vibration or other field hazards

While miniature columns have been successful as desktop high-resolution SEMs, introducing true portability to the SEM can push high-resolution imaging into exciting new applications. The micromachined electron beam column, with its high-performance and compact design, is an ideal platform to move in this direction.

\section{References:}

[1] L. Muray, Scanning, 33: 155-161 (2011).

[2] D. Linnarsson, J. Carpenter, B. Fubini, P. Gerde, L. L. Karlsson, D. J. Loftus, G. K. Prisk, U. Staufer, E. M. Tranfield, and W. Van Westrenen, Planet. Space Sci., vol. 74, no. 1, pp. 57-71, 2012.

[3] J. Spallas, C. Silver, L. Muray, T. Wells, and M. El Gomati, Microelectron. Eng. 83, 984-989 (2006).

[4] L. Muray, J. Spallas and D. Meisburger, Proc. SPIE 9236, Scanning Microscopies (2014).

[5] Y. Wu, D. Klyachko, S. Davilla, J. Spallas, S. Indermuehle, and L. Muray, J. of Vac. Sci. \& Technol. B 32 (2014).
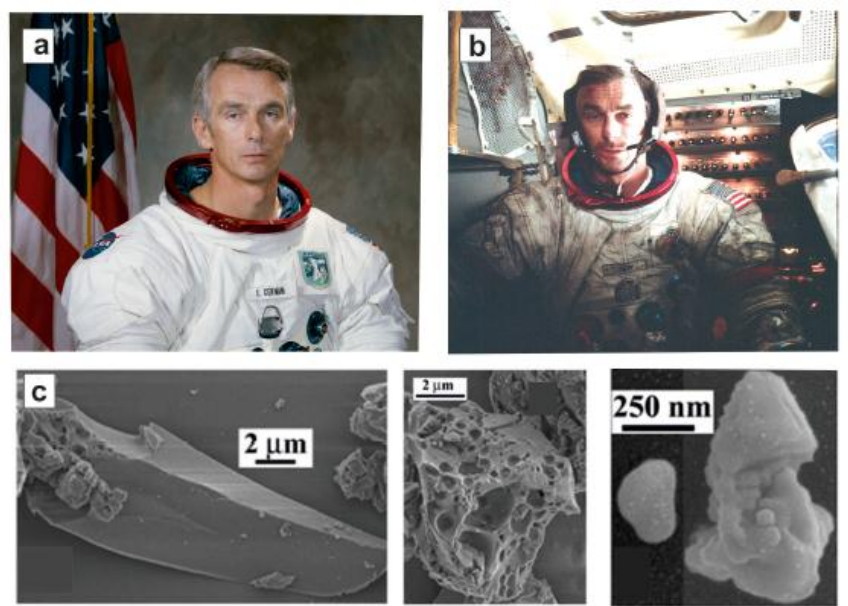

Figure 1. Lunar dust toxicity: a) Jene Cernan in a clean space suit b) suit after moon walk and c) SEM images of inhalable lunar dust

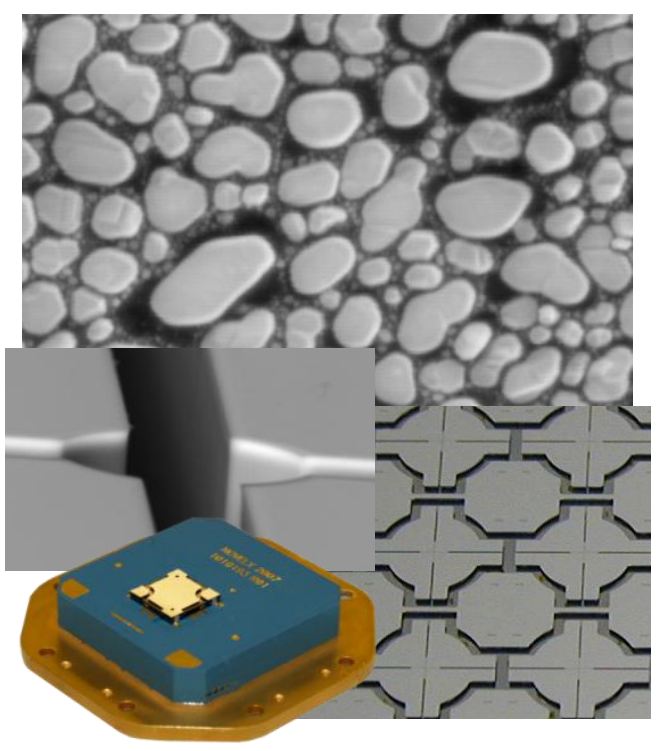

Figure 2. Miniature electron beam column 